\title{
Caracterización de egresados de la licenciatura en Biología de la FEC-LUZ (1991-2000)
}

\author{
Characterization of professional in biology degree in FEC-LUZ (1991-2000)
}

\section{Zaith Coromoto Villegas Fuentes \\ zaithvillegasfuentes@gmail.com Código ORCID: 0000-0001-6861-4699}

Universidad del Zulia, Venezuela

\author{
a- Mayerly Lisseth Nava de García \\ mayeliset18@gmail.com \\ Código ORCID: 0000-0002-0286-0229
}

Universidad del Zulia, Venezuela

\author{
a. Neyla Coromoto Ortiz Navarro \\ ncon_fico@yahoo.com \\ Código ORCID: 0000-0002-4651-5857
}

Universidad del Zulia, Venezuela

Artículo recibido en febrero 2020 | Arbitrado en marzo 2020 | Publicado en mayo 2020

\section{Resumen}

La caracterización de egresados se requiere para realizar su seguimiento, el cual es fundamental para actualizar los planes de estudio, entre otros propósitos. Esta investigación tuvo como objetivo describir características demográficas, académicas y laborales de egresados de la Licenciatura en Biología de la FEC-LUZ, durante el período 1991-2000. La muestra fue 105 individuos y se obtuvo respuesta de $42,86 \%$ de estos. Los datos se recolectaron mediante un cuestionario, aplicado principalmente por vía electrónica. Entre los graduados predominaron el sexo femenino, la edad de 49-53 años y el estado civil casado. La mayoría de los egresados nació y reside en Venezuela; ninguno culminó el pregrado en cinco años y $75,56 \%$ ha obtenido título de postgrado. El $88,89 \%$ de los egresados labora actualmente, principalmente en relación de dependencia y desempeñándose como investigador o docente universitario. En conclusión, la caracterización de graduados puede llevarse a cabo siguiendo la metodología descrita.

\footnotetext{
Abstract

The characterization of graduates is required for tracking, which is essential to update the curriculum, among other purposes. This study aimed to describe demographic, academic and employment of professional in biology degree in FEC-LUZ, during the period 1991-2000 features. The sample size was 105 individuals and a response was obtained from $42.86 \%$ of these. The data were collected through a questionnaire, applied mainly electronic. Among the graduates were predominant female sex, age $49-53$ years and married filing status. Most graduates born and living in Venezuela; none completed the undergraduate five years and $75.56 \%$ earned graduate degree. $88.89 \%$ of graduates is working currently mainly as employee and as a researcher and university lecturer. In conclusion, graduate characterization of can be carried out following the methodology described.
}

Palabras clave:

Biología; caracterización; egresados; seguimiento de egresados; Universidad del Zulia
Keywords:

Biology; characterization; following up graduates; graduates; Universidad del Zulia 


\section{INTRODUCCIÓN}

Cada vez se exige más a las instituciones de educación superior que asuman la responsabilidad de la formación de sus graduados, por lo que su éxito se mide principalmente de acuerdo a la situación laboral y al compromiso social de los mismos. En este sentido, el seguimiento de egresados constituye una vía para llevar a cabo tal medición y para evaluar los planes de estudio. La evaluación de los programas es un elemento esencial para el mejoramiento de los servicios educativos que ofrecen las universidades, teniendo como meta el alcance de los estándares de calidad nacionales e internacionales (García et al., 2019).

Según Sánchez et al. (2010), las investigaciones de seguimiento de egresados son primordiales, puesto que su opinión y praxis permite tener conocimiento sobre la pertinencia de los pensa, la calidad de la formación recibida y el desempeño profesional. Adicionalmente, se considera que el seguimiento a graduados es una herramienta útil para la planificación educativa (Báez et al., 2008), para actualizar los planes de estudio (Vázquez, et al., 2007) y la definición de políticas regionales y nacionales (Cano, 2008).

A nivel mundial, el seguimiento de egresados constituye una tendencia importante en los centros de educación superior (García et al., 2019), incluso, muchos han procedido a su institucionalización, a fin de disponer continuamente de la información del profesional y del mercado de trabajo, logrando así un ajuste exitoso de sus diseños curriculares (Blanco, 2005). Al respecto, puede acotarse que algunas instituciones universitarias venezolanas han efectuado investigaciones de sus graduados con las que han recabado datos para la actualización curricular (Blanco, 2005;
Caligiore, 2012; Caligiore y Díaz, 2013; Bañez y García, 2019).

La Universidad del Zulia (LUZ), una de las más relevantes casas de estudio de la República Bolivariana de Venezuela, no tiene, de manera institucionalizada, un programa de seguimiento para los graduados de sus carreras, empero, ha de reconocerse que, en general, se ha tratado de adaptar los diseños curriculares de las mismas a las exigencias sociales contemporáneas.

Por su parte, la Comisión de Currículo de la Licenciatura en Biología de la Facultad Experimental de Ciencias de la Universidad del Zulia (FEC-LUZ), se ha propuesto desarrollar un seguimiento laboral a todos sus licenciados (Ortiz, 2019). Para ello, al igual que para implementar un plan de seguimiento de egresados, es menester caracterizar previamente a tales profesionales, lo cual consiste en la identificación y descripción de las cualidades que los distinguen (Guzmán et al. 2008); no obstante, solamente se ha desarrollado una investigación formal sobre caracterización de graduados, que egresaron en el período 2001-2010 (Villegas, Ortiz y Pantoja, 2015).

A fin de coadyuvar a lograr el propósito de la comisión mencionada, se realizó este estudio cuyo objetivo general fue caracterizar a los Licenciados en Biología de la FEC-LUZ graduados durante los años 1991-2000; como objetivos específicos se planteó describir las principales características demográficas, académicas y laborales de estos egresados, así como establecer la relación entre algunas de esas variables.

Según las indagaciones hechas, aparte del estudio de Villegas et al. (2015), en Venezuela no se ha publicado ninguna otra investigación concerniente al seguimiento 
de graduados de licenciaturas en biología, por lo que se considera que los resultados del presente estudio no solo podrían contribuir a la evaluación y actualización permanente del diseño curricular de la Licenciatura en Biología de la Facultad Experimental de Ciencias de la Universidad del Zulia, sino a la toma de decisiones nacionales, referentes a la formación de biólogos y a su desempeño en el mercado de trabajo.

Por último, es importante resaltar que las investigaciones sobre egresados de licenciaturas en biología son escasas a nivel mundial; algunos estudios de los que se tiene conocimiento, aparte de los citados a lo largo de este artículo, son los de Salazar (2009), Jiménez (2009) y Romero et al. (2013), sin embargo, sus resultados no son comparables a los del presente trabajo, en virtud de la diferencia existente respecto a las variables analizadas.

\section{MÉTODO}

Para esta investigación se utilizó una metodología similar a la seguida por Villegas et al. (2015).

\section{Tipo de estudio y estimación de la muestra}

Se realizó una investigación de campo a nivel descriptivo en la que la población estuvo conformada por los 143 individuos que se graduaron en la Licenciatura en Biología de la FEC-LUZ durante el período 1991-2000. La lista de egresados fue suministrada por el Sistema Automatizado de Información Académica (SADIA) de la Secretaría Docente de la Universidad del Zulia. El tamaño de la muestra fue 105 y se calculó con la fórmula propuesta por la Asociación Nacional de Universidades e
Instituciones de Educación Superior de México (ANUIES) en 1998:

$$
\mathrm{n}=\frac{\mathrm{N}}{1+\frac{\mathrm{e}^{2}(\mathrm{~N}-\mathrm{l})}{\mathrm{z}^{2} \mathrm{pq}}}
$$

Donde: $\mathrm{n}=$ tamaño de la muestra, $\mathrm{N}=$ tamaño de la población, e = error muestral $(5 \%=0,05), z=1,96$ (correspondiente $\mathrm{a}$ un nivel de confianza del 95\%), $\mathrm{p}=$ frecuencia esperada del factor a estudiar $(0,5)$ y q $=1-p$ $(0,5)$.

\section{Diseño del instrumento}

Para recopilar la información de los graduados se empleó un cuestionario con preguntas cerradas dicotómicas y de respuesta múltiple. El instrumento fue diseñado en HTML y montado en la plataforma Google Drive de Gmail por medio de un formulario. Este instrumento, cuya duración media de respuesta era aproximadamente tres minutos, estuvo conformado por los siguientes apartados: 1) Presentación de los propósitos de la investigación, compromiso de que la información suministrada sería utilizada de manera confidencial e instrucciones para el rellenado; 2) Los ítems correspondientes a las variables investigadas y 3) Agradecimiento a los consultados por su contribución con el trabajo y la reiteración del manejo confidencial de los datos proporcionados. El segundo apartado incluía las secciones "Datos personales", "Datos académicos" y "Datos laborales".

\section{Localización de los egresados y aplicación del instrumento}

Para localizar a los graduados se elaboró un directorio con sus números telefónicos y 
direcciones de correo electrónico, obteniendo estos datos de contacto por varias vías: recolección de información entre los docentes de la Licenciatura en Biología de la FEC-LUZ, comunicación personal con otros egresados de la licenciatura y establecimiento de contacto con los graduados objeto de estudio a través de Facebook. Usando la información del directorio, se envió el cuestionario por correo electrónico a grupos de 20 o menos egresados, desde una dirección con dominio institucional bajo la plataforma Gmail. Puesto que no todas las direcciones de correo electrónico de los graduados se consiguieron antes del primer envío del instrumento y otras resultaron desactualizadas, se enviaron continuamente nuevos correos electrónicos con el cuestionario a los egresados cuyas direcciones se iban encontrando.

Se trató de contactar en forma telefónica a quienes no habían contestado luego de cierto tiempo; esto, con el fin de verificar si se les había enviado el cuestionario a la dirección de correo correcta, informarles del estudio y convocarlos a participar. Posteriormente, se insistió con los graduados que aún no respondían el instrumento, reenviándolo por correo electrónico al menos dos veces más, a todos los egresados de los cuales se tenía alguna dirección de correo electrónico. Adicionalmente, se envió el cuestionario por correo en un formato de Microsoft Office Word 2010 a los graduados que no contestaron el formulario Google Drive de Gmail. Finalmente, se remitió el instrumento por la aplicación de mensajería instantánea WhatsApp a egresados que no dieron respuesta al formato de Microsoft Word.

\section{Almacenamiento y análisis de los datos}

Una vez que los consultados contestaban el formulario en la plataforma Google Drive de Gmail, los datos eran almacenados automáticamente en esta plataforma en una hoja de cálculo, que posteriormente se exportó al programa Microsoft Office Excel 2010. Por otra parte, la información de los graduados que dieron respuesta al cuestionario en el formato de Word y por WhatsApp, fue transcrita directamente en la base de datos del programa Excel. Seguidamente, se hicieron las estimaciones de porcentajes para las variables estudiadas, a fin de elaborar los respectivos gráficos y describir la relación entre algunas de estas variables.

\section{RESULTADOS}

\section{Localización de los egresados y aplicación del instrumento}

Los datos de localización (números telefónicos y direcciones de correo electrónico) fueron obtenidos para el 68,53\% de la población objeto de estudio. En una investigación anterior, realizada por Villegas et al. (2015) con egresados de la misma licenciatura en los años 2001-2010, se hallaron datos para localizar al 85,63\% de la población objetivo, resultando muy efectiva para ello la herramienta tecnológica Facebook. Igualmente, para Ortiz (2017), esta red fue provechosa para el establecimiento de puentes de información para la localización de los egresados de un instituto tecnológico; no obstante, en el presente estudio, tal herramienta no fue de mucha utilidad, lo cual pudiera deberse a su menor uso por los graduados en relación con los del estudio previo, por causa de su mayor edad. Las redes sociales suponen un material muy valioso para la investigación académica e incluso, sociólogos de la Universidad de California y de Harvard, se han valido de Facebook para diferentes análisis (Romero, 2008). De acuerdo a trabajos desarrollados con 
egresados de diversas instituciones, Facebook es uno de los medios preferidos por los graduados para recibir información (Beltrán y Guerra, 2018) y es una de las redes sociales más utilizadas por los mismos (Rivera, 2019). Por otra parte, los mecanismos de comunicación con docentes y egresados de la licenciatura, sí tuvieron efectividad para la obtención de datos de localización de los egresados, al igual que en la investigación de Villegas et al. (2015).

Entre las limitaciones para localizar a los graduados, se puede mencionar que en algunas ocasiones los números telefónicos no correspondían a la persona que se deseaba contactar o estaban fuera de servicio; en otras, los correos electrónicos eran enviados a direcciones inválidas. Indiscutiblemente, la carencia inicial de un directorio con información actualizada de los egresados, constituyó un obstáculo para su localización. Resultados parecidos fueron registrados por Báez et al. (2008), al realizar estudios de seguimiento a graduados universitarios. Cabe acotar que, además de la dificultad para localizar a los egresados, al igual que en los casos señalados por Báez et al. (2008) y White (2014), se notó la renuencia de algunas personas a responder el instrumento, aunque que se les envió la solicitud en varias oportunidades por correo electrónico.

El cuestionario fue contestado por 45 graduados (42,86\% de la muestra), teniéndose un índice medio de respuesta $(45,92 \%)$ por parte de los egresados localizables, en contraste con el índice de 63,87\% publicado por Villegas et al. (2015). A pesar de que por los momentos los resultados obtenidos no pueden extrapolarse al resto de la población objetivo (debido al porcentaje de respuesta de los graduados que conformaban la muestra), los avances logrados con este trabajo serán de mucha utilidad para el desarrollo de nuevas investigaciones sobre egresados de la Licenciatura en Biología de la Facultad Experimental de Ciencias de LUZ, especialmente en lo referido al ámbito laboral; asimismo, tales resultados pueden contribuir al mejoramiento de su diseño curricular.

Al igual que en la investigación de Villegas et al. (2015) la vía más empleada en este estudio para contestar el instrumento fue la electrónica. Del total de graduados que respondió, 62,22\% usó la plataforma Google Drive de Gmail y 2,22\% el formato diseñado en Word; el 35,56\% restante contestó el cuestionario vía WhatsApp. En la investigación desarrollada con egresados de los años 20012010, también se obtuvo el mayor porcentaje de respuesta mediante la plataforma Google Drive de Gmail y el menor con el formato en Word (Villegas et al., 2015).

La aplicación del instrumento vía electrónica resultó ventajosa por su bajo costo, por la facilidad de enviarlo a muchas personas de manera rápida e incluso simultánea y por el almacenamiento automático de la información por medio de la plantilla en Google Drive. Esta forma de aplicación de instrumentos en línea también fue reportada entre otros autores por Hernández (2017), para estudios de seguimiento a graduados universitarios.

Respecto al uso de WhatsApp, cabe destacar que los egresados que contestaron el cuestionario por esta vía, manifestaron preferirla puesto que se les hacía más sencillo manejar esa aplicación que tener acceso a las preguntas en Google Drive de Gmail o en el formato diseñado en Word. Asimismo, resalta que la receptividad de los graduados hacia esta forma de consulta fue buena, pero no tanta como cuando el instrumento se envió desde un correo institucional para ser respondido en 
línea. La aplicación WhatsApp también fue usada por Bustillos (2017), Cortés, Leyva y Organista (2017), y Correa y Oliva (2019), para la recolección de información de egresados universitarios mediante encuestas, entrevistas o cuestionarios.

\section{Caracterización de los egresados}

\subsection{Información demográfica}

\subsubsection{Sexo, edad y estado civil}

Entre los graduados que respondieron el instrumento, se observó mayor cantidad de personas del sexo femenino que del masculino $(64,44$ y $35,56 \%$, respectivamente). Este resultado es muy similar al referido por Villegas et al. (2015), pero difiere de los hallazgos de una investigación realizada por
Vermont y Rosado (2002) con egresados de una Licenciatura en Biología de una universidad mexicana, quienes encontraron una relación de tres a dos entre el número de hombres y de mujeres. La edad de los graduados se ubica actualmente en el rango de 44-63 años, teniéndose la mayor proporción para el grupo de 49-53 años (48,89\%), seguido de los grupos de 44-48, 54-58 y 59-63 años, con porcentajes de 26,67, 17,78 y 6,67\%, respectivamente. Como se observa, la mayoría de los egresados son adultos en edad productiva. Para los graduados de sexo masculino, la edad osciló entre 45 y 60 años, mientras que en el caso de las mujeres, la edad máxima fue 63 y la mínima 44. En el Gráfico 1 se muestra la distribución porcentual de egresados según el grupo etario y el sexo.

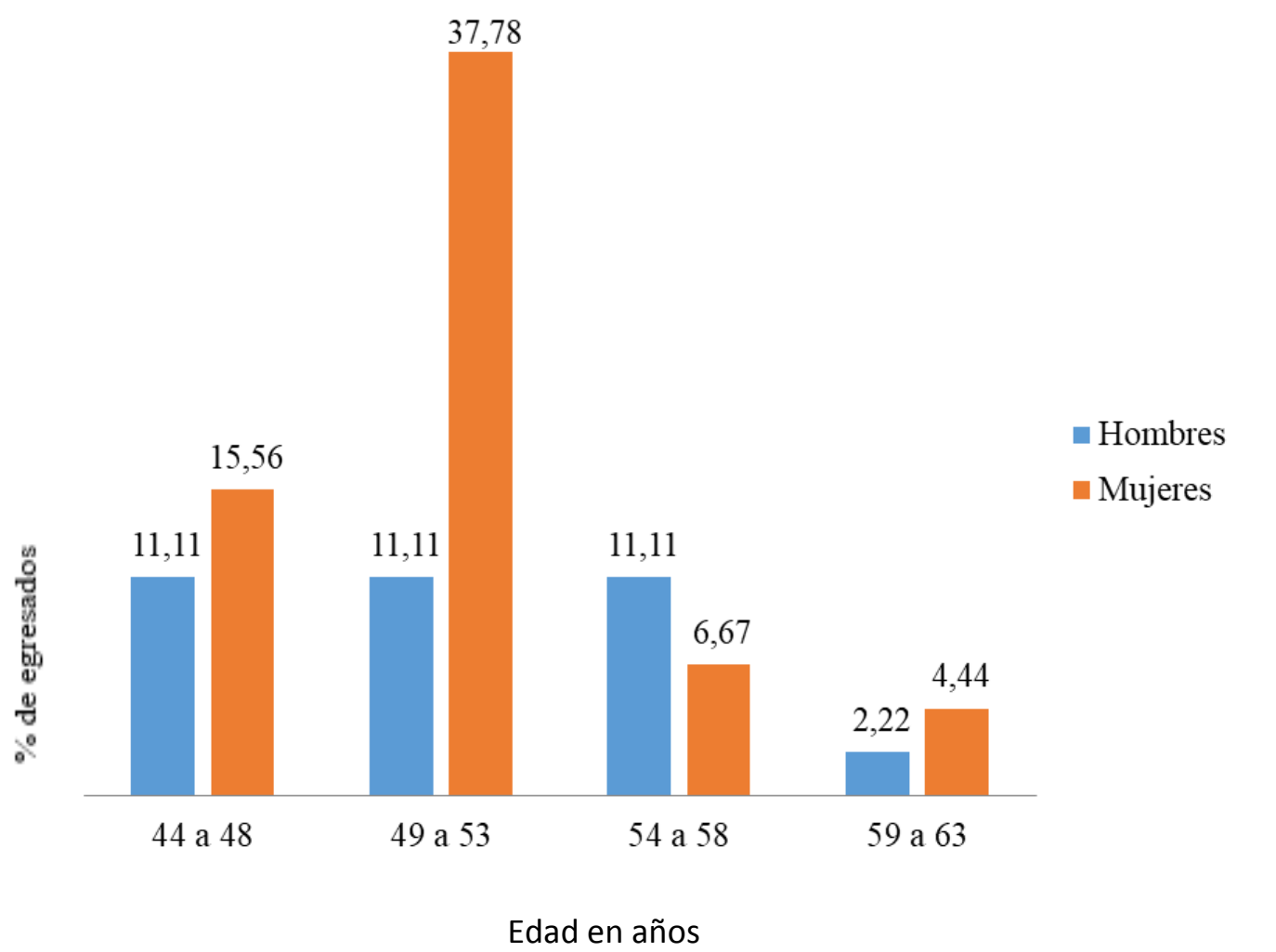

Gráfico l. Distribución porcentual de Licenciados en Biología de la Facultad Experimental de Ciencias de la Universidad del Zulia (1991-2000), según la edad y el sexo ( $\mathrm{n}=16$ hombres, 29 mujeres). 
En relación con el estado civil de los graduados, se registró que en su mayoría están casados $(60 \%)$, mientras que $31,12 \%$ son solteros, $4,44 \%$ son divorciados y $4,44 \%$ han enviudado; ninguno de ellos vive en unión libre. Al contrastar esta variable con el sexo, se obtuvo que en el caso de los hombres $75 \%$ son casados, 18,75\% solteros, 6,25\% divorciados y ninguno es viudo; respecto a las egresadas, $51,72 \%$ están casadas, $37,93 \%$ son solteras, $6,9 \%$ ha enviudado y 3,45\% son divorciadas. Se observa que para ambos sexos predominan los estados civiles casado y soltero.

\subsubsection{Lugar de nacimiento y de residencia}

Todos los egresados que contestaron el cuestionario son venezolanos, siendo principalmente oriundos del Estado Zulia (71,11\%) y, en menor proporción, del Estado Falcón (8,9\%) y del Distrito Capital $(6,67 \%)$; el resto de los graduados son originarios de los estados Trujillo, Táchira, La Guaira, Guárico, Sucre y Mérida (2,22\% de cada estado).

El 78,13\% de los graduados zulianos es originario del Municipio Maracaibo, la capital del estado, donde se halla ubicada la FEC-LUZ, 6,25\% es del Municipio Cabimas y el resto $(15,62 \%)$ nació en los municipios San Francisco, Almirante Padilla, Santa Rita, Miranda y Mara (en proporciones iguales en cada uno). Estos resultados permiten inferir, al igual que se hizo en el estudio llevado a cabo por Villegas et al. (2015), que la Licenciatura en Biología de la Facultad Experimental de Ciencias de la Universidad del Zulia tiene un carácter marcadamente regional, constituyéndose en una opción de estudio no solo para individuos provenientes de Maracaibo, sino de otros municipios cercanos a esta ciudad. Con respecto a la distribución de los egresados por país de residencia, la mayor cantidad habita en la República Bolivariana de Venezuela (82,22\%); otros informaron vivir en la República del Ecuador $(4,44 \%)$ y el resto (13,34\%) en el Canadá, los Estados Unidos de América, los Estados Unidos Mexicanos, la República Federativa del Brasil, la República Argentina y la República Libanesa (en proporciones iguales en cada país).

En cuanto al estado en el que residen los graduados que se hallan en Venezuela, $83,78 \%$ vive en el Zulia y el $16,22 \%$ restante habita, en la misma proporción, en el Distrito Capital y en los estados Miranda, Táchira Guárico, Mérida y Apure. Entre los egresados que residen en la región zuliana, $87,5 \%$ se encuentra en Maracaibo, 6,25\% en San Francisco y 6,25\% en el Municipio Miranda. Resultados similares fueron reportados por Villegas et al. (2015) en el estudio desarrollado con graduados de la Licenciatura en Biología de la FEC-LUZ en los años 2001-2010.

\subsection{Características académicas}

\subsubsection{Estudios de pregrado}

Ninguno de los egresados culminó sus estudios de pregrado en el tiempo estipulado por la universidad (cinco años) o antes; $17,78 \%$ se retrasó uno o dos años en graduarse, en tanto que 24,44\% demoró el doble o más del doble de la duración prevista (10-17 años). Un poco más de la mitad de los individuos (51,11\%), tardó ocho o nueve años en egresar y 6,67\% no suministró información en relación con esto (Gráfico 2); Villegas et al. (2015) encontraron que $92,57 \%$ de los estudiantes se graduó en más tiempo del debido. 


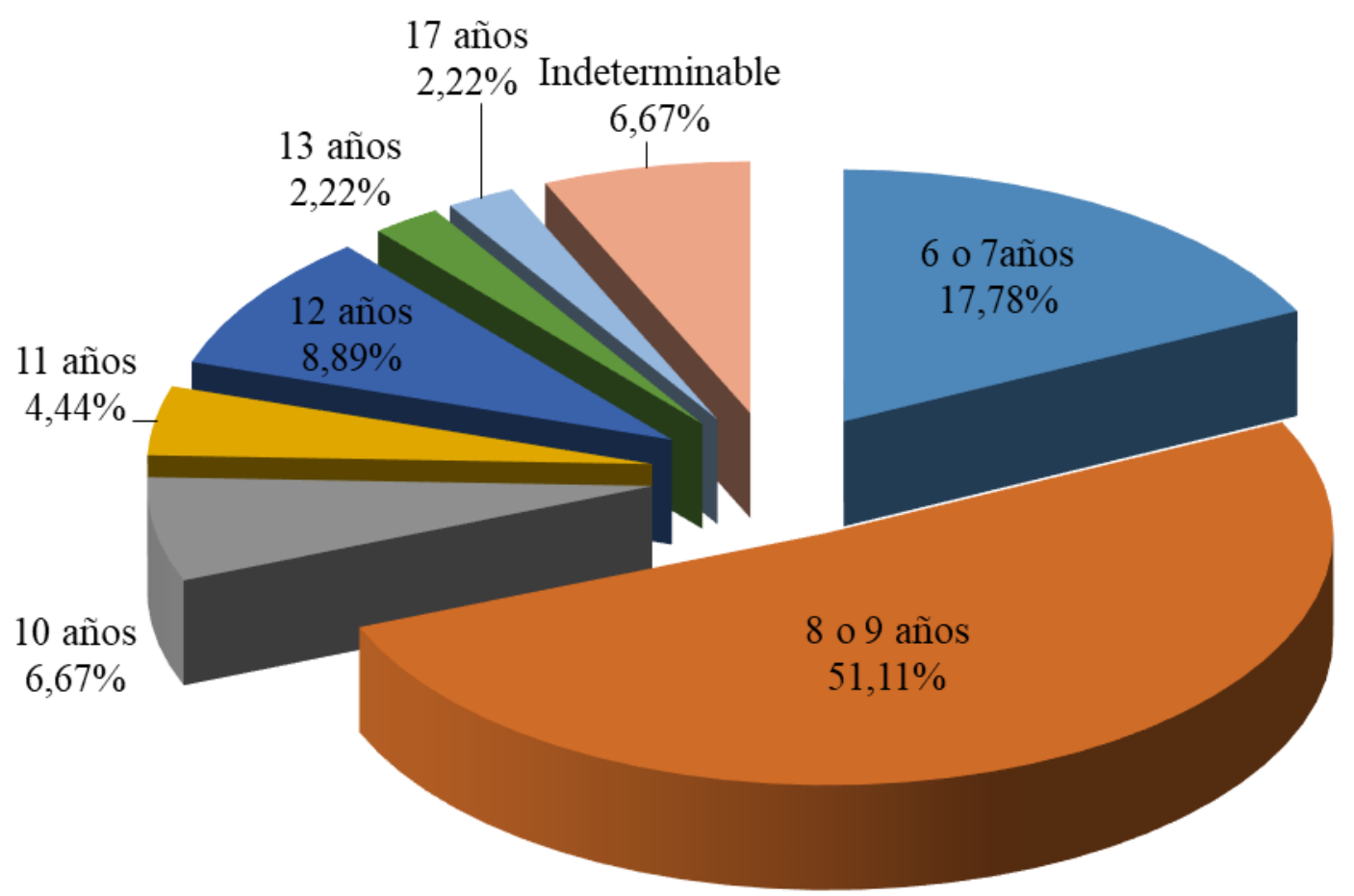

Gráfico 2. Distribución porcentual de egresados de la Licenciatura en Biología de la Facultad Experimental de Ciencias de la Universidad del Zulia (1991-2000), de acuerdo al tiempo que tardaron en culminar sus estudios ( $\mathrm{n}=45)$.

\subsubsection{Estudios de postgrado}

Solo $24,44 \%$ de los graduados no ha obtenido un título de postgrado, mientras que el resto egresó de alguna maestría $(35,56 \%)$, doctorado (11,11\%) o de ambas modalidades (28,89\%) (Gráfico 3). Estos resultados difieren de los publicados por Vermont y Rosado (2002), con respecto a Licenciados en Biología de la Universidad de Yucatán, de los cuales apenas 5,4\% de doctor; asimismo, se diferencian de lo señalado por Villegas et al. (2015), quienes determinaron que solo $35,43 \%$ de los graduados estaba titulado a nivel de postgrado, siendo el porcentaje de doctores $1,71 \%$. Es muy probable que el alto porcentaje de postgraduados registrado en la presente investigación (sobre todo de doctorado), se deba a que los consultados corresponden a promociones no recientes. El hecho de que más del 75\% de los individuos se haya seguido capacitando después de su egreso del pregrado, podría ser un indicativo de su preocupación por incrementar su capital cultural y por alcanzar una mayor competitividad en el mercado laboral. 


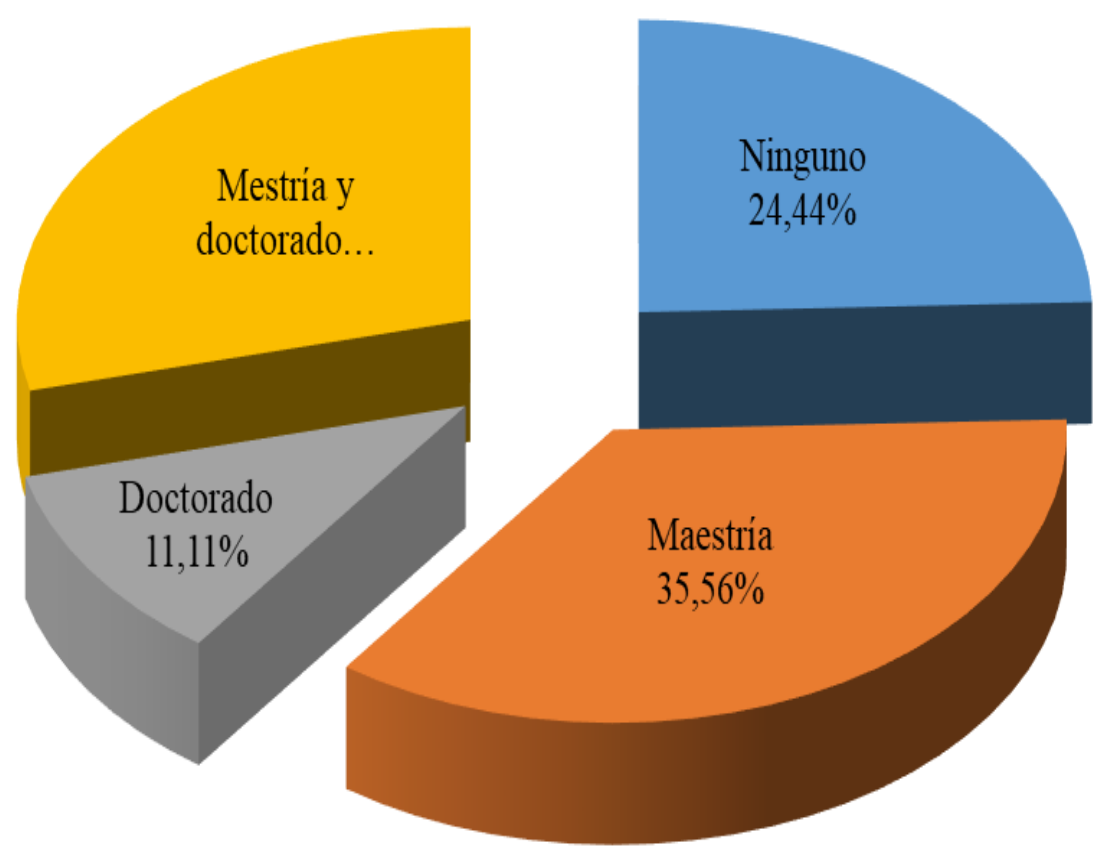

Gráfico 3. Distribución porcentual de graduados de la Licenciatura en Biología de la Facultad Experimental de Ciencias de la Universidad del Zulia (1991-2000), según el postgrado cursado (n=45).

Conforme a los datos obtenidos, la mayoría de los egresados que cursó alguna maestría escogió las áreas de ecología, microbiología e inmunología $(27,59,17,24$ y $13,79 \%$, respectivamente) $y$, en menor cantidad, las de biología celular y manejo de fauna silvestre (6,88\% en cada una). El resto de los graduados obtuvo título de magister en áreas como tecnología de los alimentos, ciencias ambientales, biotecnología vegetal, ciencias biológicas, fisiología y biofísica, proyectos de investigación, fitopatología, fruticultura e investigación educativa $(3,45 \%$ en cada área); en un caso (3,45\%), no fue suministrada la información solicitada. Tal como señalan Villegas et al. (2015), es probable que la elección por tantas personas de un postgrado en ecología o microbiología, guarde relación con el hecho de que la FECLUZ ofrece maestrías en estos campos, siendo estas opciones las únicas relacionadas directamente con el egresado.

\subsection{Información laboral}

\subsubsection{Población económicamente activa y lugar de trabajo}

El porcentaje de egresados activos laboralmente fue $88,89 \%, 6,67 \%$ se encuentra sin empleo y $4,44 \%$ ya se jubiló. La tasa de desocupación para las mujeres fue superior a la de los hombres (Gráfico 4). 


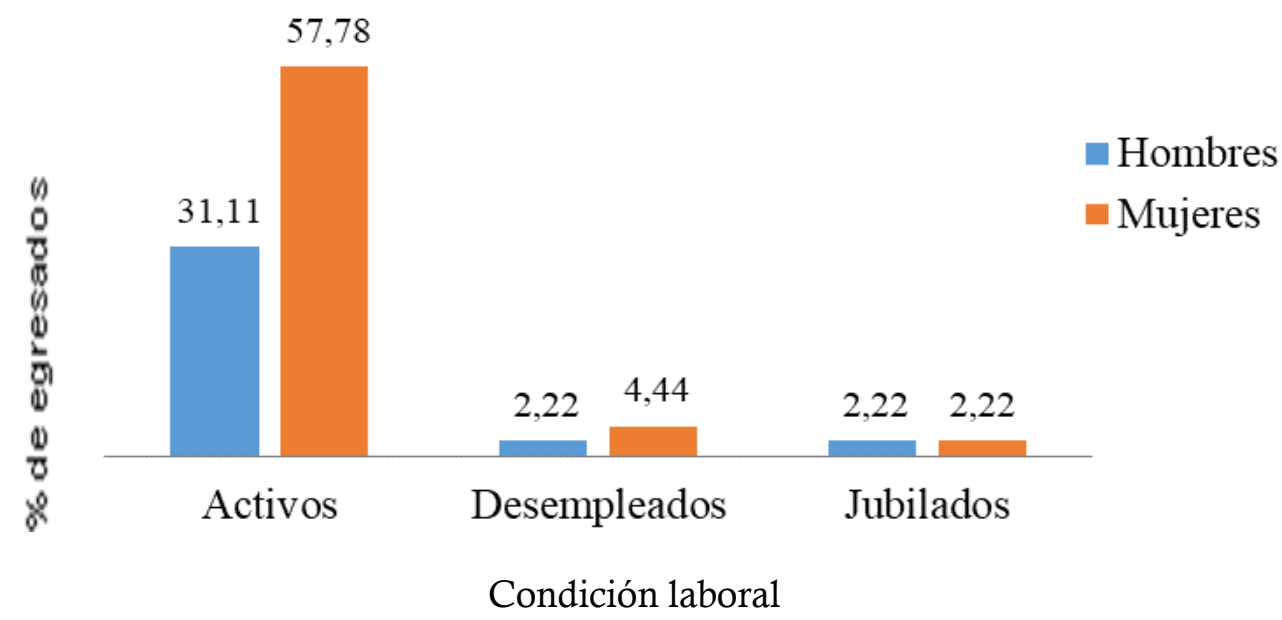

Gráfico 4. Distribución porcentual de egresados de la Licenciatura en Biología de la Facultad Experimental de Ciencias de la Universidad del Zulia (1991-2000), de acuerdo al sexo y a la condición laboral (hombres n=16, mujeres $n=29$ ).

De los graduados que se encuentran trabajando en Venezuela (82,5\% del total de los económicamente activos), 79,41\% labora en el Zulia y el resto en los estados Falcón (5,88\%), Miranda (5,88\%), y Táchira, Guárico y Apure (a razón de aproximadamente 2,94\% en cada entidad federal). Se notó que la mayor parte de los egresados trabaja en las ciudades capitales del país (60\%), sobre todo en Maracaibo y, en menor proporción, en otras localidades de Venezuela pertenecientes al Zulia y a otros estados.

Como dato interesante, se puede mencionar que el $100 \%$ de los graduados con título de postgrado se encuentra económicamente activo, lo que pudiera apoyar la premisa de que culminar este tipo de estudios, le otorga al profesional más competitividad e incrementa sus posibilidades laborales.

\subsubsection{Tipo de relación laboral y régimen jurídico de la institución o empresa de trabajo}

De los egresados económicamente activos 95\% está empleado y 5\% se desempeña de forma independiente (un graduado como modista y otro como desarrollador web/webmaster freelancer). Respecto al régimen jurídico de la empresa o institución de trabajo, se registró que $81,58 \%$ labora en instituciones públicas y $18,42 \%$ en empresas o instituciones privadas (Gráfico 5). Se coincide con Villegas et al (2015), en que los resultados revelan la importancia del sector público venezolano como fuente de trabajo para los graduados de la Licenciatura en Biología de la Facultad Experimental de Ciencias de la Universidad del Zulia, sin desestimar, por supuesto, al sector privado. 


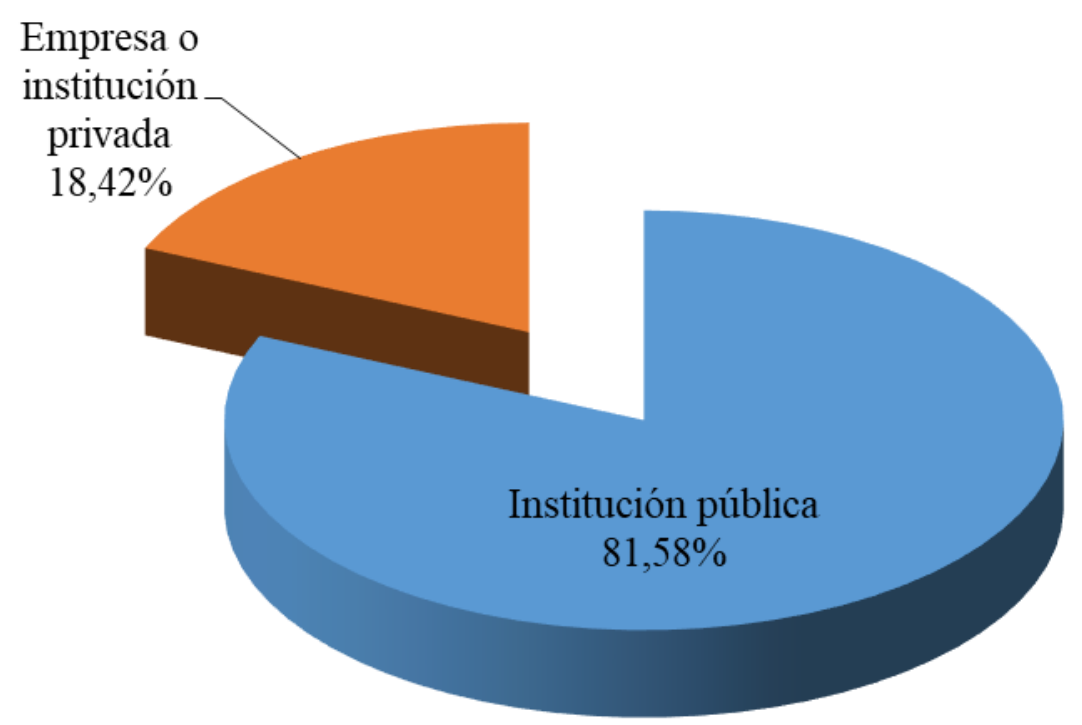

Gráfico 5. Distribución porcentual de graduados de la Licenciatura en Biología de la Facultad Experimental de Ciencias de la Universidad del Zulia (1991-2000), según el régimen jurídico de la institución o empresa donde trabajan $(\mathrm{n}=38)$.

En cuanto a la relación de las variables régimen jurídico y sexo, se tuvo que $66,67 \%$ de los hombres está laborando en instituciones públicas y $33,33 \%$ en el sector privado, mientras que para las mujeres, las cifras fueron 80,77 y 19,23\%, respectivamente. Estos resultados, al igual que todos los anteriores referidos a la información laboral, son comparables a los reportados por Villegas et al. (2015).

\subsubsection{Cargo desempeñado}

Tal como se observa en el Gráfico 6, de los egresados económicamente activos, $15 \%$ ejerce el cargo de investigador en instituciones públicas no universitarias de Venezuela (relacionadas con la generación de conocimiento y el ambiente), pero en su mayoría (65\%) laboran como docentes o investigadores en instituciones de educación superior venezolanas y extranjeras, tanto públicas como privadas. Es probable que esto último se deba a que los graduados de la Licenciatura en Biología de la FEC-LUZ, son reconocidos por tener una cultura de investigación científica, la cual es muy valorada en las universidades. 


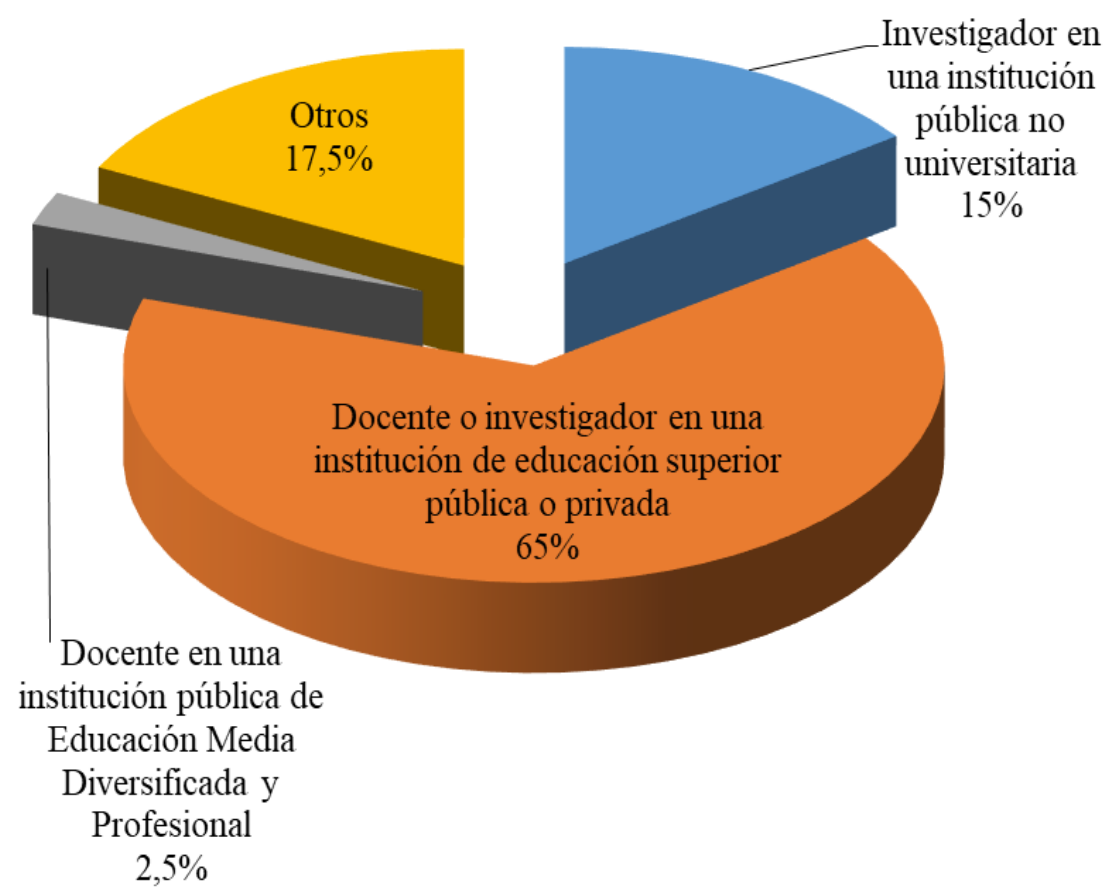

Gráfico 6. Distribución porcentual de egresados de la Licenciatura en Biología de la Facultad Experimental de Ciencias de la Universidad del Zulia (1991-2000), de acuerdo al cargo desempeñado ( $\mathrm{n}=40)$ (Otros: jefe de departamento de planta de tratamiento de aguas residuales y agua potable, asesor técnico, supervisor de laboratorio, jefe de producción, modista, desarrollador web/webmaster freelancer, jefe de laboratorio y consultor).

El cargo de docente en el nivel de Educación Media Diversificada y Profesional es ocupado por un solo egresado $(2,5 \%)$ que labora en una institución pública del Estado Zulia (Gráfico 6). Los cargos considerados por los graduados dentro de categoría Otros (17,5\%), se especifican en el Gráfico 6 y son desempeñados en su mayoría en Venezuela, en diversos lugares como una universidad pública y empresas privadas dedicadas al ramo alimenticio, el impacto ambiental y la salud. No se registró el ejercicio de egresados como investigadores independientes ni como inspectores de control de calidad.

Los resultados de esta investigación corresponden con el de Villegas et al. (2015) en relación con el hecho de que los cargos más desempeñados por los graduados son el de investigador en una institución no universitaria y el de docente o investigador en una institución de educación superior. La información reunida permite corroborar que 92,5\% de los individuos activos laboralmente ocupan cargos acordes a su perfil profesional y el $100 \%$ de los participantes en el estudio ha ejercido algún trabajo relacionado con el perfil profesional del egresado en Biología.

CONCLUSIONES

La caracterización de graduados de la Licenciatura en Biología de la FEC-LUZ puede llevarse a cabo siguiendo la metodología descrita en este estudio, siendo de efectividad para ello, el uso de la aplicación WhatsApp y, sobre todo, de la herramienta tecnológica Google Drive de Gmail. En relación a las características demográficas de los egresados durante los años 1991-2000, se determinó que la mayor proporción es del sexo femenino, del grupo etario 49-53 años, están casados y nacieron y residen en el Estado Zulia, Venezuela. De acuerdo a la información 
académica obtenida, ninguno de los graduados culminó la licenciatura en el tiempo establecido por la universidad (cinco años), pero alrededor del $75 \%$ de los mismos continuó su capacitación, obteniendo un título de postgrado en las modalidades maestría, doctorado o ambas. Referente a las características laborales, se conoció que los graduados son económicamente activos en su mayoría, laboran principalmente como empleados de instituciones públicas y ocupan cargos acordes a su perfil profesional.

\section{REFERENCIAS}

Asociación Nacional de Universidades e Instituciones de Educación Superior (ANUIES) (1998). Esquema básico para estudios de egresados en Educación Superior. México: Dirección de Servicios Editoriales

Báez, J., Morales, J., y Acosta, A. (2008). Estudio de seguimiento a egresados del programa de especialización en auditoría de la salud de la Corporación Universitaria del Caribe-CECAR período 2000-2007. Revista Investigación y Pedagogía,1(2), 46-75

Bañez, A., y García, G. (2019). Características de la transición al primer empleo. Caso: egresados de la Universidad Católica Andrés Bello en el año 2013. Revista sobre Relaciones Industriales y Laborales, (52), 6-34. Recuperado de http://revistasenlinea.saber.ucab.edu.ve/t emas/index.php/rrii2/article/viewFile/43 $83 / 3629$

Beltrán, V., y Guerra, M. (2018). Diseño de una campaña de divulgación durante el II periodo del año 2017 acerca de los beneficios y servicios que la Universidad de los Llanos ofrece a los egresados (pasantía para obtener el título de pregrado). Universidad de los Llanos, Villavicencio, Colombia
Blanco, Y. (2005). Diseño de un programa de seguimiento al egresado de la Licenciatura en Bioanálisis como contribución al rediseño del perfil profesional vigente de la carrera en la Universidad de Oriente, Núcleo Bolívar. Universidad de Oriente, Consejo de Estudios de Postgrado en educación, Núcleo Bolívar. Venezuela. Recuperado de http://postgradoeducacio nudobolivar.files.wordpress.com/2008/03/ ytalia_blanco_06.pdf el 2 de diciembre de 2012

Bustillos, D. (2017). Modelo para medir el grado de satisfacción de los egresados del Tecnológico Boliviano Alemán de la ciudad de Sucre (tesis de maestría). Universidad Andina Simón Bolívar, Sucre, Bolivia

Caligiore, I. (2012). Pertinencia de la formación académica de la carrera de enfermería. Educere, 16(54), 211-19. Recuperado de https://www.redalyc.org/pdf/356/356261 60021.pdf

Caligiore, I., y Díaz, J. (2013). Competencias en la atención primaria en salud de los egresados de la carrera de enfermería: Universidad de Los Andes. Revista ciencia y cuidado, 10(2), 40-49. Recuperado de https://dialnet.unirioja.es/servlet/articulo ?codigo $=4698068$

Cano, C. (2008). Determinantes en la eficiencia en la producción de educación. Revista de la Educación Superior, 37(147), 1730. Recuperado de http://www.scielo.org.mx/scielo.php?scr ipt $=$ sci_arttext\&pid=S0185 27602008000300002

Correa, J., y Oliva, L. (2019). Egresados en psicología opinan sobre su formación en competencias investigativas. Revista Electrónica de Psicología Iztacala,22(3), 2750-63. Recuperado de https://www.medigraphic.com/cgi$\mathrm{bin} / \mathrm{new} /$ resumen.cgi?IDARTICULO=89 688

Cortés, G., Leyva, C., y Organista, D. (2017). Comprensión de las prácticas fonoaudiológicas 
realizadas por los egresados del Programa de Fonoaudiología de la Corporación Universitaria Iberoamericana con la aplicación del modelo sistémico de la comunicación (tesis de pregrado). Corporación Universitaria Iberoamericana, Bogotá, Colombia

García, C., Treviño, A., y Banda, F. (2019). Caracterización del seguimiento de egresados universitarios. Revista Estudios del Desarrollo Social: Cuba y América Latina, 7(1), 23-38. Recuperado de file:///C:/Users/Zaith/Desktop/ART\%C3 $\% 8 D C U L O \% 20 E G R E S A D O S \% 209120$ 00/Caracterizaci\%C3\%B3n\%20del\%20s eguimiento $\% 20 \mathrm{de} \% 20$ egresados $\% 20$ uni versitarios $\% 20$ Garc $\% C 3 \% A D a \% 20 y \% 20$ col\%202019.html

Guzmán, S., Febles, M., Corredera, A., Flores, P., Tuyub, A., y Rodríguez, P. (2008). Estudio de seguimiento de egresados: recomendaciones para su desarrollo. Innovación Educativa, 8(42), 1931. Recuperado de https://www.redalyc.org/articulo.oa?id=1 79421234003

Hernández, M. (2017). Seguimiento a graduados y egresados de las promociones 2013-2014, Facultad Multidisciplinaria de Ilobasco, Universidad Católica de El Salvador. Anuario de Investigación, 6, 113-122. Recuperado de http://docplayer.es/148144913-

Seguimiento-a-graduados-y-egresadosde-las-promociones-facultadmultidisciplinaria-de-ilobascouniversidad-catolica-de-el-salvador.html Jiménez, M. (2009). Trayectorias laborales y movilidad de los biólogos agropecuarios de la Universidad Autónoma de Tlaxcala. Perfiles Educativos, 31(126), 56-79. Recuperado de http://www.scielo.org.mx/pdf/peredu/v3 $\ln 126 / v 3 \ln 126 a 4 . p d f$

Ortiz, J. (2017). Seguimiento a egresados del Tecnológico Augusto C. Sandino, INATEC, Nueva Guinea en las diferentes modalidades técnicas agropecuarias (tesis de maestría). Universidad Nacional Autónoma de Nicaragua, Juigalpa, Nicaragua

Ortiz, N. (2019). Comunicación personal. Universidad del Zulia. Facultad Experimental de Ciencias. Departamento de Biología. Maracaibo, Venezuela

Rivera, M. (2019). Estrategias de comunicación con el egresado de la Tecnología en Gestión del Talento Humano del Centro de Comercio del SENA a partir de un modelo de seguimiento e interacción (Tesis de maestría). Institución Universitaria Esumer, Medellín, Colombia

Romero, P. (2008). El insaciable apetito de las redes sociales. El Mundo. Recuperado de https://www.elmundo.es/navegante/200 8/01/18/tecnologia/1200676010.html

Romero, M., Rodríguez, C., y Martínez, Y. (2013). Satisfacción laboral de egresados de la Universidad Autónoma de Sinaloa: estudio de ocho carreras. Revista Electrónica de Investigación en Educación Superior, 1(1), 1-13

Salazar, C. (2009). Seguimiento de Graduados de la Escuela de Biología de la Facultad de Ciencias Químicas y Farmacia de la Universidad de San Carlos de Guatemala (tesis de maestría). Universidad Mariano Gálvez, Guatemala Sánchez, L., Gutiérrez, E., Valdez, M., Sánchez, I., y Reyna, J. (2010). Sistema de Información para el Seguimiento de Egresados de las Maestrías del Instituto Tecnológico de Aguascalientes EGRESATEC. Conciencia Tecnológica, (40), 20-24. Recuperado de https://www.redalyc.org/articulo.oa?id=9 4415759005

Vázquez, E., Rojo, A., y Gómez, N. (2007). Seguimiento de 62 Pediatras Egresados del Hospital Infantil del Estado de Sonora (HIES). Bol Clin Hosp Infant Edo Son, 24(2), 66-72. Recuperado de https://pdfs.semanticscholar.org/f30b/72 0840b526f60a99fb8f76bb846844e90fe9. pdf 
Vermont, R., y Rosado, C. (2002). Seguimiento de egresados de la Licenciatura en Biología de la Facultad de Medicina Veterinaria y Zootecnia de la UADY. Nueva época, 6(11), 87-97. Recuperado de https://pdfs.semanticscholar.org/2831/8c 08d9c0b88ff3bf9c06bd96f9d438afelb5.p df?_ga=2.230918111.1109205695.15879069 97-2103383105.1587906997

Villegas, Z., Ortiz, N., y Pantoja, J. (2015). Caracterización de egresados de la Licenciatura en Biología de la Facultad Experimental de Ciencias de la Universidad del Zulia (2001-2010). Omnia (1), 9-23. Recuperado de https://www.redalyc.org/pdf/737/737421 21002.pdf

White, H. (2014). El componente educativo en las trayectorias profesionales de los egresados de la Maestría en Antropología de la Universidad de Los Andes (tesis de maestría). Universidad de Los Andes, Bogotá, Colombia

\section{AGRADECIMIENTOS}

Esta investigación estuvo enmarcada dentro del proyecto $\mathrm{CH}-0418-16$, avalado por el Consejo de Desarrollo Científico, Humanístico y Tecnológico de la Universidad del Zulia (CONDES-LUZ). 